\title{
KMT-2019-BLG-0842Lb: A Cold Planet below the Uranus/Sun Mass Ratio
}

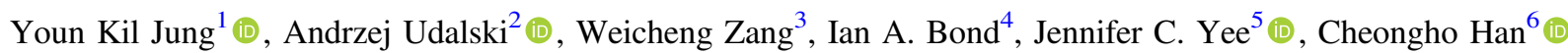
and

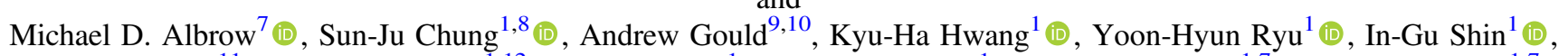

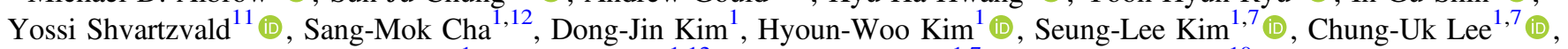
Dong-Joo Lee ${ }^{1}$, Yongseok Lee ${ }^{1,12}$, Byeong-Gon Park ${ }^{1,7}$, Richard W. Pogge ${ }^{10}$ (it)

(KMTNet Collaboration),

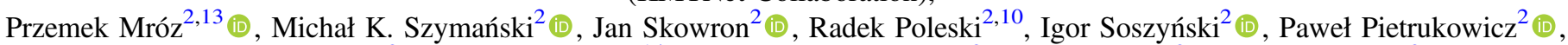
Szymon Kozłowski ${ }^{2}$, Krzysztof Ulaczyk ${ }^{14}\left({ }^{1}\right.$, Krzysztof A. Rybicki ${ }^{2}$, Patryk Iwanek ${ }^{2}$ (D), Marcin Wrona ${ }^{2}$

(OGLE Collaboration),

and

Fumio Abe ${ }^{15}$, Richard Barry ${ }^{16}$ (1), David P. Bennett ${ }^{16,17}$ (1), Aparna Bhattacharya ${ }^{16,17}$, Martin Donachie ${ }^{18}$, Hirosame Fujii ${ }^{15}$, Akihiko Fukui ${ }^{19,20}$ (1), Yuki Hirao ${ }^{21}$, Yoshitaka Itow ${ }^{15}$ (1) , Yukei Kamei $^{15}$, Iona Kondo ${ }^{21}$ (i), Naoki Koshimoto ${ }^{22,23}$ (1), Man Cheung Alex Li ${ }^{18}$, Yutaka Matsubara ${ }^{15}$, Shota Miyazaki ${ }^{21}$ (1), Yasushi Muraki ${ }^{15}$, Masayuki Nagakane ${ }^{21}$, Clément Ranc ${ }^{16}$ (1), Nicholas J. Rattenbury ${ }^{18}$ (D), Yuki Satoh ${ }^{21}$, Hikaru Shoji ${ }^{21}$, Haruno Suematsu ${ }^{6}$, Denis J. Sullivan ${ }^{24}$, Takahiro Sumi ${ }^{21}$, Daisuke Suzuki $^{25}$ (D) Paul J. Tristram ${ }^{26}$, Takeharu Yamakawa ${ }^{15}$, Tsubasa Yamamwaki ${ }^{21}$, and Atsunori Yonehara ${ }^{27}$

(The MOA Collaboration)

${ }^{1}$ Korea Astronomy and Space Science Institute, Daejeon 34055, Republic of Korea

${ }^{2}$ Astronomical Observatory, University of Warsaw, Al. Ujazdowskie 4, 00-478 Warszawa, Poland

${ }^{3}$ Department of Astronomy and Tsinghua Centre for Astrophysics, Tsinghua University, Beijing 100084, People's Republic of China

${ }_{5}^{4}$ Institute of Natural and Mathematical Science, Massey University, Auckland 0745, New Zealand

${ }^{5}$ Center for Astrophysics | Harvard \& Smithsonian, 60 Garden St., Cambridge, MA 02138, USA

${ }^{6}$ Department of Physics, Chungbuk National University, Cheongju 28644, Republic of Korea

${ }^{7}$ University of Canterbury, Department of Physics and Astronomy, Private Bag 4800, Christchurch 8020, New Zealand

${ }^{8}$ Korea University of Science and Technology, Korea, (UST), 217 Gajeong-ro, Yuseong-gu, Daejeon, 34113, Republic of Korea

${ }^{9}$ Max-Planck-Institute for Astronomy, Königstuhl 17, D-69117 Heidelberg, Germany
${ }^{10}$ Department of Astronomy, Ohio State University, 140 W. 18th Ave., Columbus, OH 43210, USA

${ }^{11}$ Department of Particle Physics and Astrophysics, Weizmann Institute of Science, Rehovot 76100, Israel

${ }^{12}$ School of Space Research, Kyung Hee University, Yongin, Kyeonggi 17104, Republic of Korea

${ }^{13}$ Division of Physics, Mathematics, and Astronomy, California Institute of Technology, Pasadena, CA 91125, USA

${ }^{14}$ Department of Physics, University of Warwick, Gibbet Hill Road, Coventry, CV4 7AL, UK

${ }^{15}$ Institute for Space-Earth Environmental Research, Nagoya University, Nagoya 464-8601, Japan

${ }^{16}$ Code 667, NASA Goddard Space Flight Center, Greenbelt, MD 20771, USA

${ }^{17}$ Department of Astronomy, University of Maryland, College Park, MD 20742, USA

${ }^{18}$ Department of Physics, University of Auckland, Private Bag 92019, Auckland, New Zealand

${ }^{19}$ Department of Earth and Planetary Science, Graduate School of Science, The University of Tokyo, 7-3-1 Hongo, Bunkyo-ku, Tokyo 113-0033, Japan

${ }^{20}$ Instituto de Astrofísica de Canarias, Vía Láctea s/n, E-38205 La Laguna, Tenerife, Spain

${ }^{21}$ Department of Earth and Space Science, Graduate School of Science, Osaka University, Toyonaka, Osaka 560-0043, Japan

${ }^{22}$ Department of Astronomy, Graduate School of Science, The University of Tokyo, 7-3-1 Hongo, Bunkyo-ku, Tokyo 113-0033, Japan

${ }^{23}$ National Astronomical Observatory of Japan, 2-21-1 Osawa, Mitaka, Tokyo 181-8588, Japan

${ }^{24}$ School of Chemical and Physical Science, Victoria University, Wellington, New Zealand

${ }^{25}$ Institute of Space and Astronautical Science, Japan Aerospace Exploration Agency, Kanagawa 252-5210, Japan

${ }^{26}$ University of Canterbury Mt. John Observatory, P.O. Box 56, Lake Tekapo 8770, New Zealand

${ }^{27}$ Department of Physics, Faculty of Science, Kyoto Sangyo University, Kyoto 603-8555, Japan

Received 2019 December 8; revised 2020 September 6; accepted 2020 September 16; published 2020 November 10

\begin{abstract}
We report the discovery of a cold planet with a very low planet/host mass ratio of $q=(4.09 \pm 0.27) \times 10^{-5}$, which is similar to the ratio of Uranus/Sun $\left(q=4.37 \times 10^{-5}\right)$ in the solar system. The Bayesian estimates for the host mass, planet mass, system distance, and planet-host projected separation are $M_{\text {host }}=0.76 \pm 0.40 M_{\odot}$, $M_{\text {planet }}=10.3 \pm 5.5 M_{\oplus}, D_{\mathrm{L}}=3.3 \pm 1.3 \mathrm{kpc}$, and $a_{\perp}=3.3 \pm 1.4 \mathrm{au}$, respectively. The consistency of the color and brightness expected from the estimated lens mass and distance with those of the blend suggests the possibility that the most blended light comes from the planet host, and this hypothesis can be established if high-resolution images are taken during the next (2020) bulge season. We discuss the importance of conducting optimized photometry and aggressive follow-up observations for moderately or very high magnification events to maximize the detection rate of planets with very low mass ratios.
\end{abstract}

Unified Astronomy Thesaurus concepts: Gravitational microlensing (672); Gravitational microlensing exoplanet detection (2147)

Supporting material: data behind figure 


\section{Introduction}

For the first 15 years of microlensing planet detections, there was no clear evidence for cold planets (i.e., beyond the snow line) with mass ratios below that of Uranus $\left(q=4.37 \times 10^{-5}\right)$ neither in our solar system nor in other systems. There are of course several cold, low-mass bodies in the outer solar system, but these are more than 10 times less massive than the smallest terrestrial planet (Mercury). The same applies to Ceres, which lies approximately on the snow line. Such bodies likely form by a distinct mechanism relative to planets, and they were therefore reclassified as dwarf planets by the IAU.

Cold, low-mass planets in exosystems can only be detected by microlensing (Bennett \& Rhie 1996; Beaulieu et al. 2006; Gould et al. 2006). However, there was no discovery of such a planet until one with a mass ratio of $q=4.7 \times 10^{-5}$ was reported in 2017 (Udalski et al. 2018). ${ }^{28}$

In principle, the lack of such detections could have been due to one of the following four causes: (1) poor sensitivity of microlensing searches to such planets; (2) extreme rarity (or complete absence) of such planets in nature; (3) adverse fluctuation of small number statistics; or (4) some combination of these. Suzuki et al. (2016) showed that there was a break in the power-law distribution (in $q$ ) below some threshold, estimated roughly at $q_{\mathrm{br}} \sim 1.7 \times 10^{-4}$, arguing in particular that their Microlensing Observations in Astrophysics (MOA) based planet sample had significant sensitivity below the lowest $q$ detections. Udalski et al. (2018) confirmed that if the mass ratio distribution of planets with $q<1 \times 10^{-4}$ were modeled as a power law, its slope was rising with $q$, in contrast to the well-established falling power law at higher $q$. Moreover, they showed that four of the seven planets that they analyzed would have been detected even if their mass ratios had been below $q=3 \times 10^{-5}$, and one of these would have been detected even at $q=2 \times 10^{-6}$, i.e., below the Earth/Sun mass ratio. Jung et al. (2019) argued that the dearth of planets below $q=4.7 \times 10^{-5}$ was due either to a break in the mass-ratio function at $q_{\mathrm{br}} \simeq 5.6 \times 10^{-5}$ or a pile-up of planets near the Neptune-mass-ratio threshold.

With a mass ratio of $q=(1.8 \pm 0.2) \times 10^{-5}$, KMT-2018BLG-0029 was the first reported planet that lay clearly below the previous apparent Uranus/Sun mass-ratio threshold (Gould et al. 2020). It provided proof of the existence of such planets, but a single detection yielded very limited information on their frequency. Gould et al. (2020) reviewed the history of the (by then, nine) $q<1 \times 10^{-4}$ planet detections, and concluded that with the advent of regular observations of the Korea Microlensing Telescope Network (KMTNet; Kim et al. 2018b, 2018c, 2018d) in 2016, the rate of such detections would be increasing, and that it should be possible to probe the frequency of such low-mass planets within a few years.

Although microlensing is sensitive to planets with mass ratios below $q \sim 10^{-4}$, the number of known microlensing planets with very low mass ratios is still small and these planets comprise a very minor fraction of all microlensing planets. Therefore, detecting more planets in the low-mass regime is important to investigate the physical parameter distributions of these planets

\footnotetext{
${ }^{28}$ Within their sample of seven $q<1 \times 10^{-4}$ microlensing planets, OGLE2016-BLG-1195 had the lowest mass ratio of $q=(4.7 \pm 0.5) \times 10^{-5}$. Udalski et al. (2018) took the average from two groups who had made independent parameter determinations using disjointed data sets: $q=$ $[(4.2 \pm 0.7)$ and $(5.5 \pm 0.8)] \times 10^{-5}$ from Bond et al. (2017) and Shvartzvald et al. (2017), respectively. Udalski et al. (2018) excluded OGLE-2017-BLG0173 from their sample because its solution was ambiguous by a factor of 2.5 $(2.48 \pm 0.24$ versus $6.4 \pm 1.0) \times 10^{-5}$ at $\Delta \chi^{2}=3.5$ (Hwang et al. 2018)
}

and to compare the distributions with those of planets in the higher mass regime. In this paper, we report the detection of a cold planet with a mass ratio of $q=(4.09 \pm 0.27) \times 10^{-5}$.

\section{Observations}

KMT-2019-BLG-0842, (R.A., decl. $)_{\mathrm{J} 2000}=(17: 53: 50.03$, $-29: 52: 38.78)$, corresponding to $(l, b)=(+0.11,-2.02)$, was discovered by the KMT alert-finder system (Kim et al. 2018a) and announced as a microlensing candidate on the KMTNet website ${ }^{29}$ at UT 02:39 on 2019 May 16, about six days before the event reached its peak. The event was independently found by the Optical Gravitational Lensing Experiment (OGLE; Udalski et al. 2015) and MOA (Bond et al. 2004) collaborations at UT 19:30 on 2019 May 18 as OGLE2019-BLG-0763 and at UT 07:02 on 2019 May 24 as MOA2019-BLG-232, respectively.

Observations by the KMTNet survey were conducted utilizing three identical $1.6 \mathrm{~m}$ telescopes equipped with $\left(2^{\circ} \times 2^{\circ}\right)$ cameras, located at Cerro Tololo InterAmerican Observatory (KMTC), the South African Astronomical Observatory (KMTS), and the Siding Springs Observatory (KMTA; Kim et al. 2016). KMT-2019-BLG-0842 lies in the overlapping KMT fields BLG02 and BLG42, each of which is observed at a nominal cadence of $\Gamma=2 \mathrm{hr}^{-1}$. However, both fields were observed at an adjusted cadence of $\Gamma=3 \mathrm{hr}^{-1}$ from KMTS and KMTA prior to June 15, i.e., until more than three weeks after the peak of the event. Hence, the effective cadence was $\Gamma=4 \mathrm{hr}^{-1}$ from KMTC and $\Gamma=6 \mathrm{hr}^{-1}$ from KMTS and KMTA. The observations were primarily conducted in the $I$ band, but every tenth such observation was matched by a $V$ band observation in order to measure the source color.

The OGLE collaboration observed the event, located in their BLG501 field, with $\Gamma=1 \mathrm{hr}^{-1}$ cadence using the $1.3 \mathrm{~m}$ telescope, equipped with a $1.4 \mathrm{deg}^{2}$ camera, located at Las Campanas Observatory in Chile. OGLE observations were also primarily conducted in the $I$ band.

The light curve derived from the KMTNet and OGLE observations is shown in Figure 1. The KMTNet data were reduced using pySIS (Albrow et al. 2009), which is a variant of difference image analysis (DIA; Tomaney \& Crotts 1996; Alard \& Lupton 1998; Alard 2000). The OGLE data were reduced using another variant of DIA (Woźniak 2000). The relatively brief $(\sim 15 \mathrm{hr})$ planetary anomaly was announced by $\mathrm{W}$. Zang at UT 19:56 on 2019 May $26\left(\mathrm{JD}^{\prime}=\mathrm{JD}-\right.$ $2450000=8630.33$ ), but by this time the anomaly was already over, and thus no follow-up observations of the anomaly resulted from the announcement.

The MOA collaboration observed the object with a cadence of $\Gamma=4 \mathrm{hr}^{-1}$ using its $1.8 \mathrm{~m}$ telescope at Mt. John, New Zealand, which is equipped with a $2.2 \mathrm{deg}^{2}$ camera. However, observational conditions were poor on the night of the anomaly. The resulting observations therefore do not constrain the characteristics of the planet, although they do qualitatively confirm its existence. We therefore do not include these data in the primary fit, but rather use them to illustrate this confirmation (Figure 2). MOA observations were mainly taken using a broad $R / I$ filter.

\footnotetext{
${ }^{29}$ http://kmtnet.kasi.re.kr/ ulens
} 

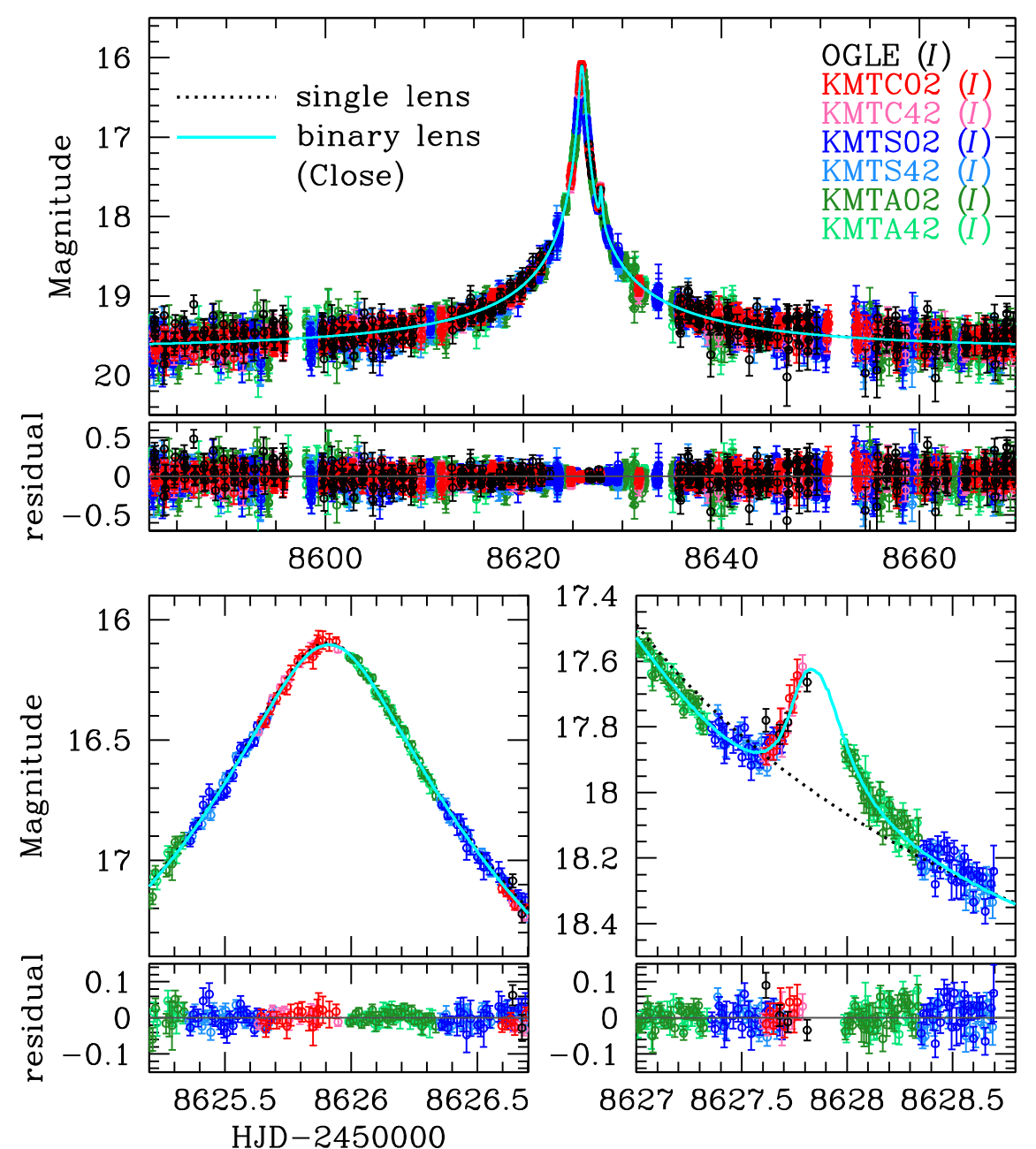

Figure 1. Light curve and close 2L1S model for KMT-2019-BLG-0842. Zooms of the peak and the anomaly are shown in the lower left and lower right panels, respectively. The anomaly lasts about 15 hours despite the very low planet-host mass ratio $q=4.1 \times 10^{-5}$ because the anomaly occurs $\sim 7 \times t_{\text {eff }}$ effective timescales after peak, implying that the trajectory is at an acute angle of $\alpha \simeq \cot ^{-1} 7 \sim 8.5$ relative to the planet-host axis.

(The data used to create this figure are available.)

\section{Light Curve Analysis}

With the exception of the short anomaly peaking at $t_{0, \text { anom }}=$ 8627.85, KMT-2019-BLG-0842 has the general appearance of a Paczyński (1986) single-lens single-source (1L1S) event. The light curve of a $1 \mathrm{~L} 1 \mathrm{~S}$ event is characterized by three geometric parameters $\left(t_{0}, u_{0}\right.$, and $\left.t_{\mathrm{E}}\right)$, respectively the time of maximum, the impact parameter (in units of the Einstein radius $\theta_{\mathrm{E}}$ ), and the Einstein radius crossing time,

$$
\begin{gathered}
t_{\mathrm{E}} \equiv \frac{\theta_{\mathrm{E}}}{\mu_{\text {rel }}} ; \quad \theta_{\mathrm{E}}^{2} \equiv \kappa M \pi_{\mathrm{rel}} \\
\kappa \equiv \frac{4 G}{c^{2} \mathrm{AU}} \simeq 8.14 \frac{\mathrm{mas}}{M_{\odot}},
\end{gathered}
$$

where $M$ is the lens mass and $\left(\pi_{\text {rel }}, \boldsymbol{\mu}_{\text {rel }}\right)$ are the lens-source relative parallax and proper motion, respectively. From visual inspection, $t_{0} \simeq 8625.91$ and the FWHM of the light curve is 1.0 days. The peak magnification is either moderately high or very high depending on the blending, which is difficult to estimate by eye, and in either case $u_{0} \ll 1$, so $t_{\text {eff }} \equiv u_{0} t_{\mathrm{E}}=$ FWHM/ $\sqrt{12} \simeq 0.29$ day. Hence (e.g., Gould \& Loeb 1992), the source almost certainly passes at an angle of

$$
\alpha=\tan ^{-1} \frac{t_{\text {eff }}}{t_{0, \text { anom }}-t_{0}}=\tan ^{-1} 0.149=0.148,
$$

or 8.5 , relative to the binary axis. The fact that the anomaly is very short, even though the source crosses the caustic structure at such an acute angle $\alpha$, suggests that the mass ratio between the binary lens components is very low. A similar inference can be drawn from the fact that there is no noticeable anomaly over the high (or very high) magnification peak. That is, both effects tend to indicate a small, resonant (or near-resonant) caustic. However, detailed modeling is required to proceed further.

\subsection{Binary Lens (2L1S) Analysis}

We model the light curve as a $2 \mathrm{~L} 1 \mathrm{~S}$ event with seven nonlinear parameters $\left(t_{0}, u_{0}, t_{\mathrm{E}}, s, q, \alpha\right.$, and $\left.\rho\right)$, where $s$ is the projected separation between the binary components (normalized to $\left.\theta_{\mathrm{E}}\right), \rho \equiv \theta_{*} / \theta_{\mathrm{E}}$, and $\theta_{*}$ is the angular source radius. Notwithstanding the above estimate of $\alpha$, we conduct a broad grid search in the parameters $s, q$, and $\alpha$, in which $(s, q)$ are 


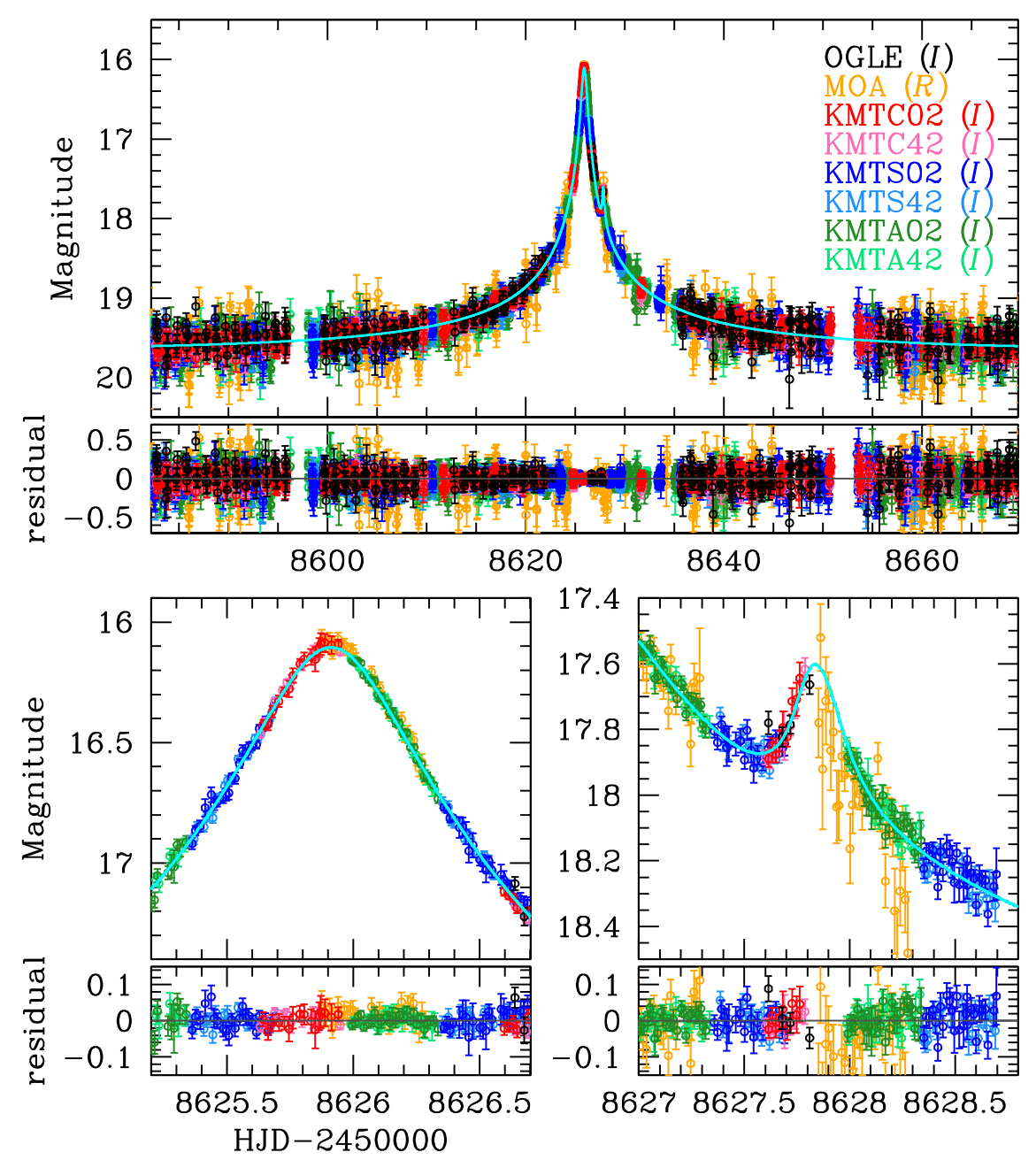

Figure 2. Light curve KMT-2019-BLG-0842, as shown in Figure 1, but with MOA data aligned to the best-fit model. The MOA data confirm the anomaly but do not contribute to the constraining of the model and so are not included in the fit.

held fixed and the remaining five parameters are allowed to vary in a Markov Chain Monte Carlo (MCMC). The three parameters $\left(t_{0}, u_{0}\right.$, and $\left.\mathrm{t}_{\mathrm{E}}\right)$ are seeded at their Paczyński (1986) values, $\rho$ is seeded at $10^{-3}$, and $\alpha$ is seeded at six values drawn uniformly from the unit circle. There are two flux parameters $\left(f_{s, i}, f_{b, i}\right)$ for each observatory $i$, which are fit by linear regression to the observed flux $F_{i}(t)$ for each model, according to $F_{i}(t)=f_{s, i} A(t)+f_{b, i}$. These are identified with the source and blend flux, respectively.

We find only two local minima in the resulting $(s, q)$ map, which we then further refine by allowing all seven parameters to vary in the MCMC. Finally, these converge to $(s, q)=$ $\left(0.98,4.1 \times 10^{-5}\right)$ and $(s, q)=\left(1.06,3.9 \times 10^{-5}\right)$. See Table 1 . These solutions are actually quite similar in all of their parameters, and in particular both agree with the analytically estimated value of the source trajectory angle $\alpha$ in Equation (2). However, the wide solution $(s>1)$ is disfavored relative to the close solution $(s<1)$ by $\Delta \chi^{2}=176$. Furthermore, the wide solution has clear systematic residuals. See Figure 3. Hence, we exclude the wide solution. The caustic geometries for both the close and wide solutions are shown in Figure 4.

We investigate whether the microlens parallax vector $\pi_{\mathrm{E}}$ (Gould 1992, 2000, 2004) can be meaningfully constrained, but we find that it cannot.
Table 1

Binary Lens Models

\begin{tabular}{lcc}
\hline \hline Parameters & Close & Wide \\
\hline$\chi^{2} /$ dof & $10065.4 / 10332$ & $10241.5 / 10332$ \\
$t_{0}\left(\mathrm{HJD}^{\prime}\right)$ & $8625.914 \pm 0.010$ & $8625.914 \pm 0.011$ \\
$u_{0}\left(10^{-3}\right)$ & $6.565 \pm 0.202$ & $5.393 \pm 0.183$ \\
$t_{\mathrm{E}}($ days $)$ & $43.859 \pm 1.287$ & $52.915 \pm 1.708$ \\
$s$ & $0.983 \pm 0.013$ & $1.063 \pm 0.013$ \\
$q\left(10^{-5}\right)$ & $4.079 \pm 0.289$ & $3.901 \pm 0.249$ \\
$\alpha(\mathrm{rad})$ & $0.146 \pm 0.067$ & $0.144 \pm 0.072$ \\
$\rho\left(10^{-4}\right)$ & $4.246 \pm 0.966$ & $0.282_{-0.090}^{+0.913}$ \\
$f_{\mathrm{s}}$ & 0.037 & 0.030 \\
$f_{\mathrm{b}}$ & 0.179 & 0.185 \\
\hline
\end{tabular}

Note. HJD $^{\prime}=$ HJD -2450000 days.

\subsection{Binary Source (1L2S) Analysis}

Gaudi (1998) pointed out that a short-lived bump on an otherwise normal Paczyński (1986) light curve could in principle be produced by a second source (1L2S) rather than a second lens (2L1S). This is of particular concern when (as in the present case) the bump does not exhibit any obvious caustic structure. Therefore, we check the degeneracy between the 


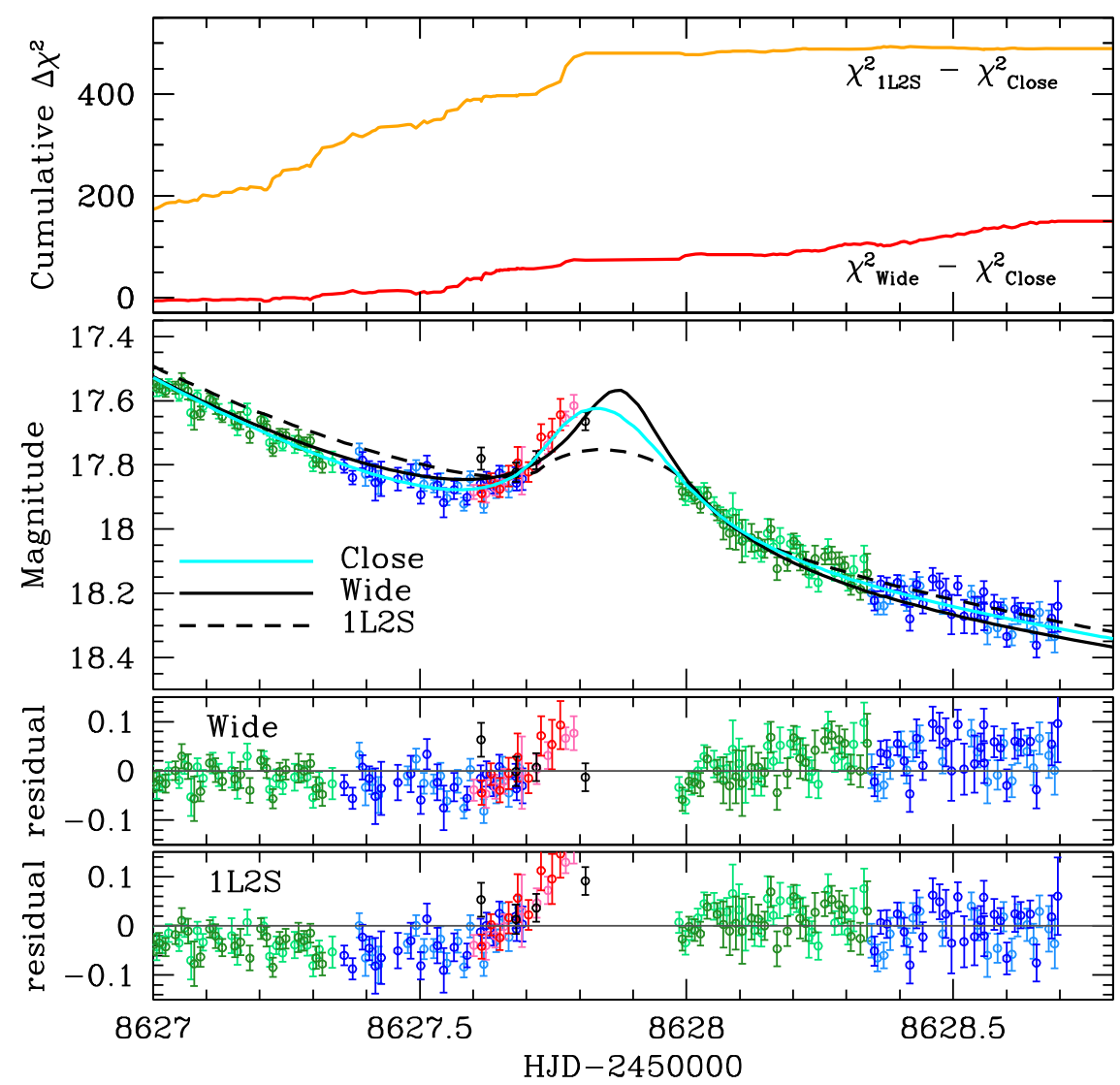

Figure 3. Upper panel: $\Delta \chi^{2}$ difference relative to the only surviving model (2L1S close) of two other possible models, $2 \mathrm{~L} 1 \mathrm{~S}$ wide (red) and $1 \mathrm{~L} 2 \mathrm{~S}$ (yellow) for KMT2019-BLG-0842. The second panel shows the anomaly region of the light curve together with these three models. The lower two panels show the residuals for the two excluded models. See Figure 1 for the corresponding residuals of the surviving (2L1S close) model.

2L1S and 1L2S interpretations by additionally conducting a 1L2S modeling. Our 1L2S model has eight nonlinear parameters: $2 \times\left(t_{0}, u_{0}, \rho\right)$ for the two sources, plus a common Einstein timescale $t_{\mathrm{E}}$ and the $I$-band flux ratio between the two sources, $q_{F, I}$. See Table 2.

We find that the $2 \mathrm{~L} 1 \mathrm{~S}$ interpretation is favored over the $1 \mathrm{~L} 2 \mathrm{~S}$ interpretation by $\Delta \chi^{2}=\chi^{2}(1 \mathrm{~L} 2 \mathrm{~S})-\chi^{2}(2 \mathrm{~L} 1 \mathrm{~S})=495$. To check the region of the fit difference, we also inspect the cumulative $\Delta \chi^{2}$ distribution of $\Delta \chi^{2}=\chi^{2}(1 \mathrm{~L} 2 \mathrm{~S})-$ $\chi^{2}(2 \mathrm{~L} 1 \mathrm{~S})$ (see Figure 3). From this, we find that the $\chi^{2}$ difference largely comes from the anomaly region, in which the 1L2S solution provides a poorer fit to the observed light curve, especially in the rising part of the anomaly. In addition, the solution is unphysical in the sense that the flux ratio is $q_{F, I} \simeq 0.013$ while the normalized sources sizes $\left(\rho_{1}, \rho_{2}\right)$ are of the same order. ${ }^{30}$ See Table 2. Hence, we exclude the 1L2S solution.

\section{Color-Magnitude Diagram and Einstein Radius}

Although the planetary anomaly does not exhibit obvious caustic features, the results in Table 1 show that the normalized source size $\rho$ is reasonably well measured. ${ }^{31}$ This implies that we can estimate $\theta_{\mathrm{E}}=\theta_{*} / \rho$ provided that $\theta_{*}$ is measured. Indeed, although the $1 \sigma$ error in $\rho$ is relatively large, there is a

\footnotetext{
${ }^{30}$ Strictly speaking, $\rho_{1}$ is poorly constrained by the fit, but it has a strict upper limit $\rho_{1}<u_{0,1}=56 \times 10^{-4}$, which implies the same unphysicality.

31 This was also the case for the low- $q$ planetary event OGLE-2016-BLG-1195 (Bond et al. 2017; Shvartzvald et al. 2017).
}

very strong, $3 \sigma$, upper limit, $\rho<5.7 \times 10^{-4}$, which will place important physical constraints on the lens system, as we discuss below.

We follow the standard approach (Yoo et al. 2004) of measuring $\theta_{*}$ by placing the source on an instrumental colormagnitude diagram (CMD). See Figure 5. For this purpose, we reduce the KMTC42 $V$ and $I$ data using pyDIA, which yields field-star and light-curve photometry on the same system. Using this instrumental system, we find source and red-clumpcentroid positions of $[(V-I), I]_{\mathrm{S}}=(2.38,21.73) \pm(0.03$, $0.01)$ and $\left[(V-I), I_{\mathrm{cl}}=(2.43,16.04) \pm(0.03,0.07)\right.$, respectively, and hence an offset of $\Delta[(V-I), I]=(-0.05$, $5.69) \pm(0.04,0.07)$. We adopt $[(V-I), \quad]_{\mathrm{cl}, 0}=(1.06$, 14.44) (Bensby et al. 2013; Nataf et al. 2013). We do not assign an error to this determination but rather add 5\% error in quadrature to the final result for all aspects of the method. We then obtain $[(V-I), I]_{\mathrm{S}, 0}=[(V-I), I]_{\mathrm{cl}, 0}+\Delta[(V-I), I]=$ $(1.01,20.13) \pm(0.04,0.07)$. Assuming that the source is located at the mean distance to the bulge, i.e., $D_{\mathrm{S}}=8.17 \mathrm{kpc}$ (Nataf et al. 2013), the source star has the color and absolute magnitude of $\left[(V-I)_{0}, M_{I}\right]_{\mathrm{S}} \simeq(1.0,5.6)$, which is typical of an early $\mathrm{K}$ dwarf.

We then convert the measured $V / I$ color into $V / K$ color using the VIK color-color relations of Bessell \& Brett (1988) and then apply the color/surface-brightness relations of Kervella et al. (2004) to obtain (after adding 5\% error)

$$
\theta_{*}=0.416 \pm 0.031 \mu \text { as. }
$$



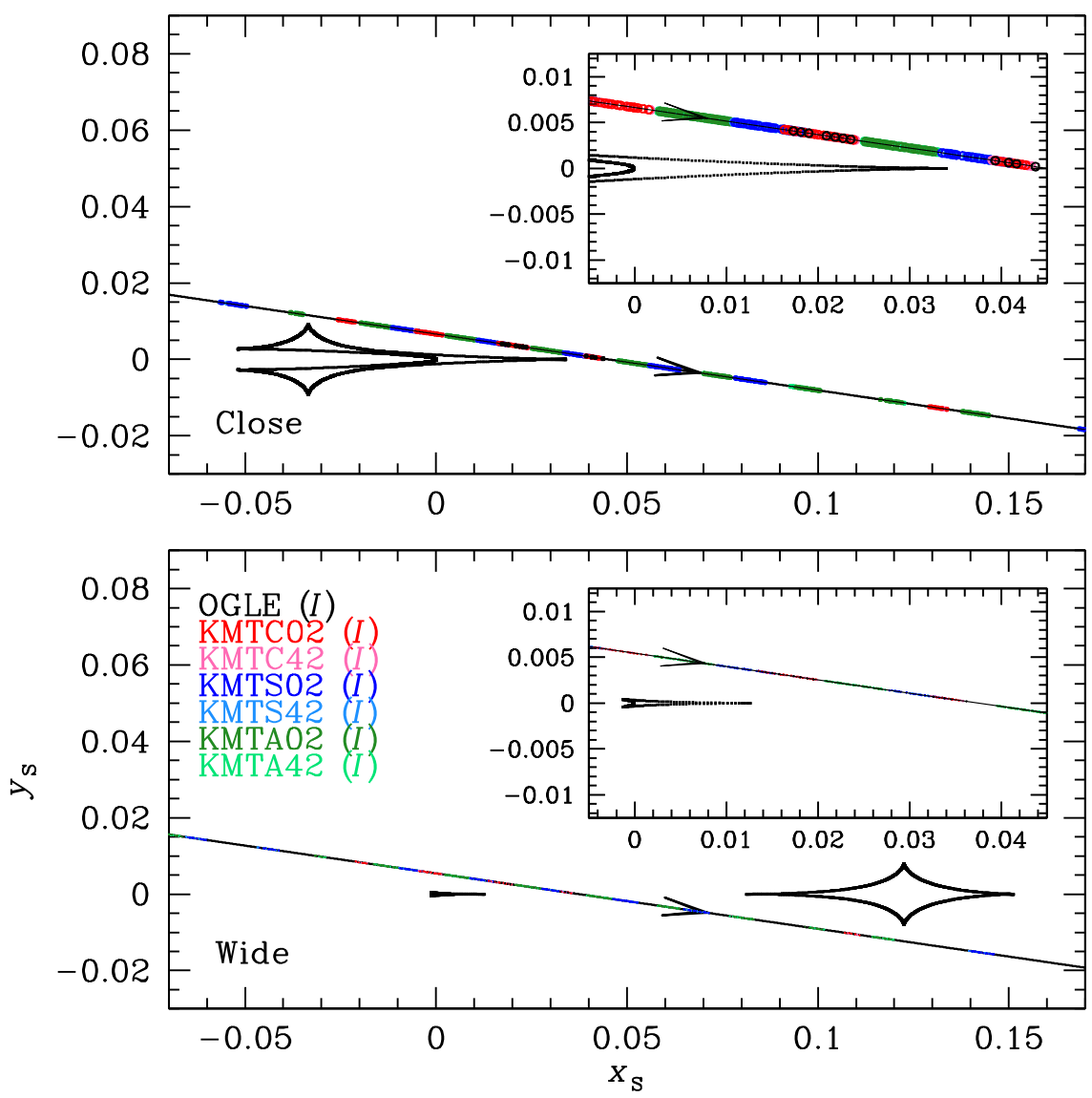

Figure 4. Caustic geometries for the two possible 2L1S solutions for KMT-2019-BLG-0842, i.e., close (top) and wide (bottom). In both cases, the anomaly (see zooms in Figures 1 and 3$)$ is generated when the source passes over the planet-star axis about 1.94 days $(\simeq 0.044$ Einstein units $)$ after the peak. In both cases, the anomaly is due to a narrow high-magnification ridge that extends from the narrow end of a caustic centered on the host. For the close geometry, this caustic has a resonant (six-sided) topology, while for the wide geometry it is a central caustic that is connected to the planetary caustic by the ridge. However, the wide geometry is excluded by $\Delta \chi^{2}=176$. See the text and Figure 3 .

Table 2

Binary Source Model

\begin{tabular}{lc}
\hline \hline Parameters & $1 \mathrm{~L} 2 \mathrm{~S}$ \\
\hline$\chi^{2} /$ dof & $10560.1 / 10031$ \\
$t_{0,1}\left(\mathrm{HJD}^{\prime}\right)$ & $8625.907 \pm 0.013$ \\
$u_{0,1}\left(10^{-3}\right)$ & $5.620 \pm 0.213$ \\
$t_{0,2}\left(\mathrm{HJD}^{\prime}\right)$ & $8627.886 \pm 0.032$ \\
$u_{0,2}\left(10^{-3}\right)$ & $0.005 \pm 0.247$ \\
$t_{\mathrm{E}}($ days $)$ & $48.657 \pm 1.710$ \\
$\rho_{1}\left(10^{-4}\right)$ & $7.6_{-1.5}^{+16.0}$ \\
$\rho_{2}\left(10^{-4}\right)$ & $32.3 \pm 2.2$ \\
$q_{F, I}\left(10^{-2}\right)$ & $1.291 \pm 0.043$ \\
$f_{\mathrm{s}}$ & 0.033 \\
$f_{\mathrm{b}}$ & 0.183 \\
\hline
\end{tabular}

We can then obtain naive estimates for $\theta_{\mathrm{E}}$ and $\mu_{\text {rel }}$,

$$
\begin{aligned}
\theta_{\mathrm{E}} & =\frac{\theta_{*}}{\rho}=0.96 \pm 0.22 \text { mas; } \\
\mu_{\text {rel }} & =8.0 \pm 1.8 \mathrm{mas} \mathrm{yr}^{-1} \quad \text { (naive). }
\end{aligned}
$$

We refer to these two estimates as naive because, as will be discussed in Section 5, the proper evaluation of these quantities should be estimated from their Bayesian posteriors.

The estimated values of $\theta_{\mathrm{E}}$ and $\mu_{\mathrm{rel}}$ strongly imply that the lens lies in the disk. That is, from the definition of $\theta_{\mathrm{E}}$
(Equation (1)), $\pi_{\text {rel }}=0.12 \operatorname{mas}\left(\theta_{\mathrm{E}} / \mathrm{mas}\right)^{2} /\left(M / M_{\odot}\right)$. Thus, if we adopt the best-fit value of $\theta_{\mathrm{E}}$ and also take account of the fact that lenses significantly more massive than the Sun would be easily visible, then we would infer $\pi_{\text {rel }} \gtrsim 0.12$ mas, i.e., a lens distance of $D_{\mathrm{L}} \lesssim 4 \mathrm{kpc}$. Because the error in $\theta_{\mathrm{E}}$ is large (due primarily to the large error in $\rho$ ), we must also consider how smaller values of $\theta_{\mathrm{E}}$ (i.e., below the best-fit value) would affect this argument. However, as mentioned above, the errors in $\rho$ are highly asymmetric, so there is a very hard lower limit, $\theta_{\mathrm{E}}>0.65$ mas. This somewhat relaxes the above argument, but still requires $\pi_{\text {rel }} \gtrsim 0.06$ mas, which puts the lens in the disk.

\section{Physical Parameter Estimates}

Because the microlens parallax $\pi_{\mathrm{E}}$ is not measured, one cannot directly infer the lens mass as $M=\theta_{\mathrm{E}} / \kappa \pi_{\mathrm{E}}$ and lenssource relative parallax as $\pi_{\mathrm{rel}}=\theta_{\mathrm{E}} \pi_{\mathrm{E}}$ from the microlensing data. We therefore conduct a Bayesian analysis by incorporating priors from a Galactic model. In fact, the situation is somewhat more complicated than usual because the measurement error of the normalized source radius $\rho$ is large and its distribution is asymmetric. Moreover, $\rho$ is correlated with the planet-host mass ratio $q$. Thus, in sharp contrast to the usual case, the posterior estimate of this (seemingly) pure microlensing-light-curve parameter $q$ is actually affected by the Galactic priors. 


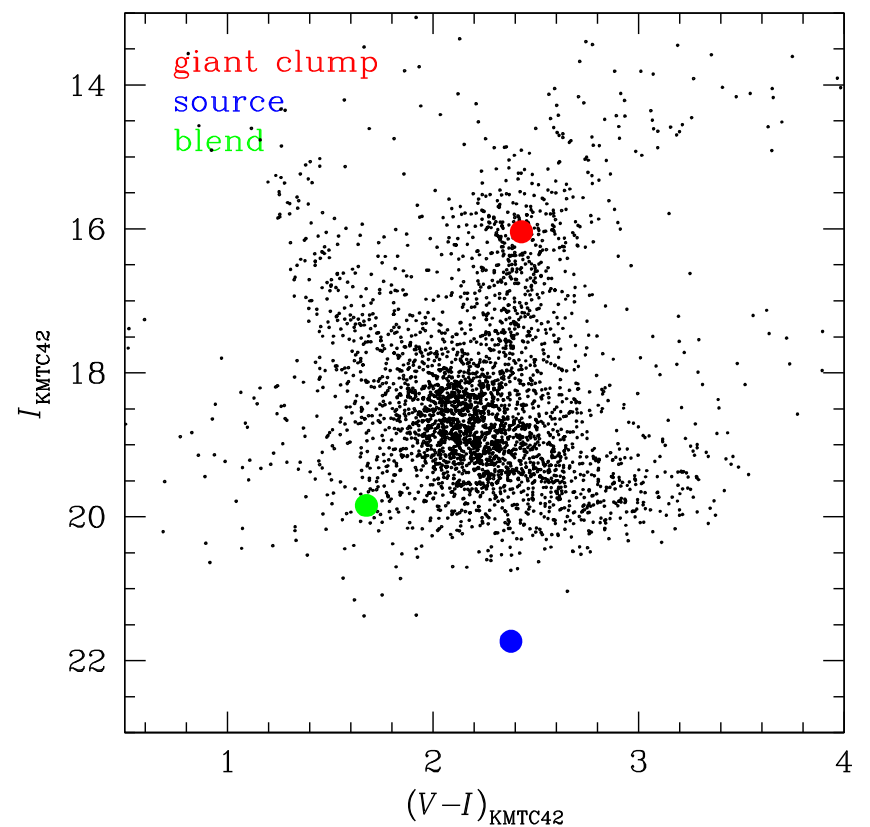

Figure 5. Color-magnitude diagram (CMD) for field stars in a $2^{\prime}$ square centered on KMT-2019-BLG-0842 derived from pyDIA reductions of KMTC42 data. The positions of the source (blue), the blend (green), and the centroid of the red clump (red) are indicated. The error bars for these determinations are discussed in the text. In particular, the blend color is relatively poorly determined. By cross-matching to the calibrated OGLE-III catalog (Udalski et al. 2008; Szymanski et al. 2011), we find $I_{\text {calib }}=$ $I_{\mathrm{KMTC} 42 \mathrm{C}}-0.085,(V-I)_{\mathrm{calib}}=(V-I)_{\mathrm{KMTC} 42 \mathrm{C}}-0.140$.

The fundamental features of the Galactic model and the Bayesian procedures are the same ${ }^{32}$ as those presented in Jung et al. (2018). Here, we focus on describing what is different for the present case.

As usual, we incorporate constraints of both $t_{\mathrm{E}}$ and $\theta_{\mathrm{E}}$ determined from the light-curve modeling and CMD. For this, we weigh the simulated events by $W_{t_{\mathrm{E}}}=\exp \left[-\left(t_{\mathrm{E}}-t_{\mathrm{E} . \text { best }}\right)^{2} / 2 \sigma_{t_{\mathrm{E}}}^{2}\right]$, where $t_{\mathrm{E}}$ is the Einstein timescale of the simulated event and $\left(t_{\mathrm{E} . b e s t}, \sigma_{t_{\mathrm{E}}}\right)$ are the values from Table 1.

We incorporate the $\theta_{\mathrm{E}}$ constraint in a different way from that of the $t_{\mathrm{E}}$ constraint. We first evaluate a function $\Delta \chi^{2}(\rho)$ by running MCMCs for a series of fixed values of $\rho$. See Figure 6. Then, for each simulated event, we evaluate $\theta_{\mathrm{E}}=\sqrt{\kappa M \pi_{\text {rel }}}$ from the values of $M, D_{\mathrm{L}}$, and $D_{\mathrm{S}}$ of the individual simulated events. In this process, we draw $\theta_{*}$ from a Gaussian distribution with the mean and standard deviation presented in Equation (3). We then evaluate $\rho=\theta_{*} / \theta_{\mathrm{E}}$ and finally determine the weight by $W_{\theta_{\mathrm{E}}}=\exp \left[-\Delta \chi^{2}(\rho) / 2\right]$.

The Bayesian analysis then carried out following a usual procedure, but with one major exception. In Figure 6, we show the best-fit value of $q$ and its $1 \sigma$ error bar for each fixed- $\rho$ MCMC, that is carried out following the procedure described above, to evaluate $\Delta \chi^{2}(\rho)$. Over the region of principal interest, $3 \lesssim \rho / 10^{-4} \lesssim 5$, this shows a logarithmic (powerlaw) gradient of $d \log q / d \log \rho \simeq-0.2$. This gradient is present because the models account for the observed duration of the bump by the combination of $q$ and $\rho$ : for relatively large $q$, this duration is explained by the width of the ridge, but with decreasing $q$ it must be increasingly explained by larger $\rho$. For most events, such a gradient would play a small role because $\rho$

\footnotetext{
32 We note that Jung et al. (2018) do not specify their upper-mass cutoff for the mass function. For completeness, we note that this cutoff is $63 M_{\odot}$.
}

would be well determined. However, in the present case, the error in $\rho$ is large, so that the error in $q$ induced by this correlation is comparable to the scatter in $q$ at fixed $\rho$. Therefore, to properly evaluate the posterior value of $q$, we should incorporate the correlation into the Bayesian analysis.

We do so by evaluating $q$ separately for each simulated event in the MCMC. We first find $\rho$ (as above). We then find $q(\rho)$ and $\sigma_{q}(\rho)$ from a table that corresponds to Figure 6, and then draw $q$ randomly from a Gaussian described by these two values. This value of $q$ is then used both to evaluate the planet mass for the simulated event $m_{p}=q M_{\text {host }}$ and to find the posterior distribution of $q$ itself.

The results of the Bayesian analysis are shown in Table 3 and Figure 7. Although the lens is believed to lie in the disk from the estimated $\theta_{\mathrm{E}}$, we formally consider both bulge and disk lenses in the Bayesian analysis. As expected, we find that the chance that the lens is in the bulge is very low, $<1 \%$.

\subsection{Does the Lens Account for the Blended Light?}

The best-fit position of the blended light $[(V-I)$, $I_{b}=(1.68,19.84)$ lies at a position with offsets $\Delta[(V-I)$, $I_{b}=[(V-I), I]_{b}-[(V-I), I]_{\mathrm{cl}}=(-0.75,3.80)$ from the red clump centroid on the CMD. We discuss the error bars on this position below.

The $I$-band brightness offset of $\Delta I_{b}=3.80$ is consistent with the lens mass and distance ranges derived from the Bayesian analysis above, as summarized in Figure 7. More specifically, assuming the lens distance as the median of the posterior distance distribution, at $D_{\mathrm{L}}=3.3 \mathrm{kpc}$, the lens would be at about $z \simeq-0.1 \mathrm{kpc}$ below the galactic plane, and so plausibly behind about half the dust toward the bulge, toward which $[E(V-I)$, $\left.A_{I}\right]=(1.28,1.50)$ (Nataf et al. 2013). This would imply an absolute magnitude of the lens of $M_{I}=\left(I_{\mathrm{cl}, 0}+\Delta I\right)-A_{I} / 2-$ $[5 \log (3300)-5]=14.44+3.80-1.50 / 2-12.59 \simeq 4.9$. This corresponds roughly to an $M=0.85 M_{\odot}$ star, which is quite compatible with the Bayesian host-mass estimation. Such a star would have (depending on its metallicity) roughly $(V-I)_{0} \sim$ 0.85 , and therefore would be $\Delta(V-I)=0.85-1.06-1.28 /$ $2 \simeq-0.85$ mag bluer than the clump. Given the measurement errors (which we estimate just below), this is also quite compatible with the observed offset: -0.75 mag bluer than the clump.

The baseline object is quite faint in the $V$ band, $V_{\text {base }}=$ 21.43. It is therefore barely detected, and hence the error in magnitudes is large. The contribution from the source to this baseline light is known quite precisely, so from a conceptual point of view, we should consider the errors in the point-spread function (PSF) modeling of the remaining blended light, $V_{b}=21.52$. The error in this estimate is comprised of three distinct components: Poisson errors from finite photon statistics; systematic errors from the PSF modeling photometry program, operating under the assumption that the background is smooth; and statistical errors due to the fact that a mottled distribution of unresolved stars contributes significantly to the background.

Based on photon statistics, we estimate the systematic error as 0.19 mag. Because the PSF photometry program is relatively complex, it is difficult to reliably estimate the systematic errors. Hence, we ignore these errors for the moment. To estimate the statistical errors due to the mottled background, we apply the approach of Ryu et al. (2020), i.e., modeling the Holtzman et al. (1998) luminosity function adjusted for the local surface brightness of the bulge and the local extinction. In fact, the 


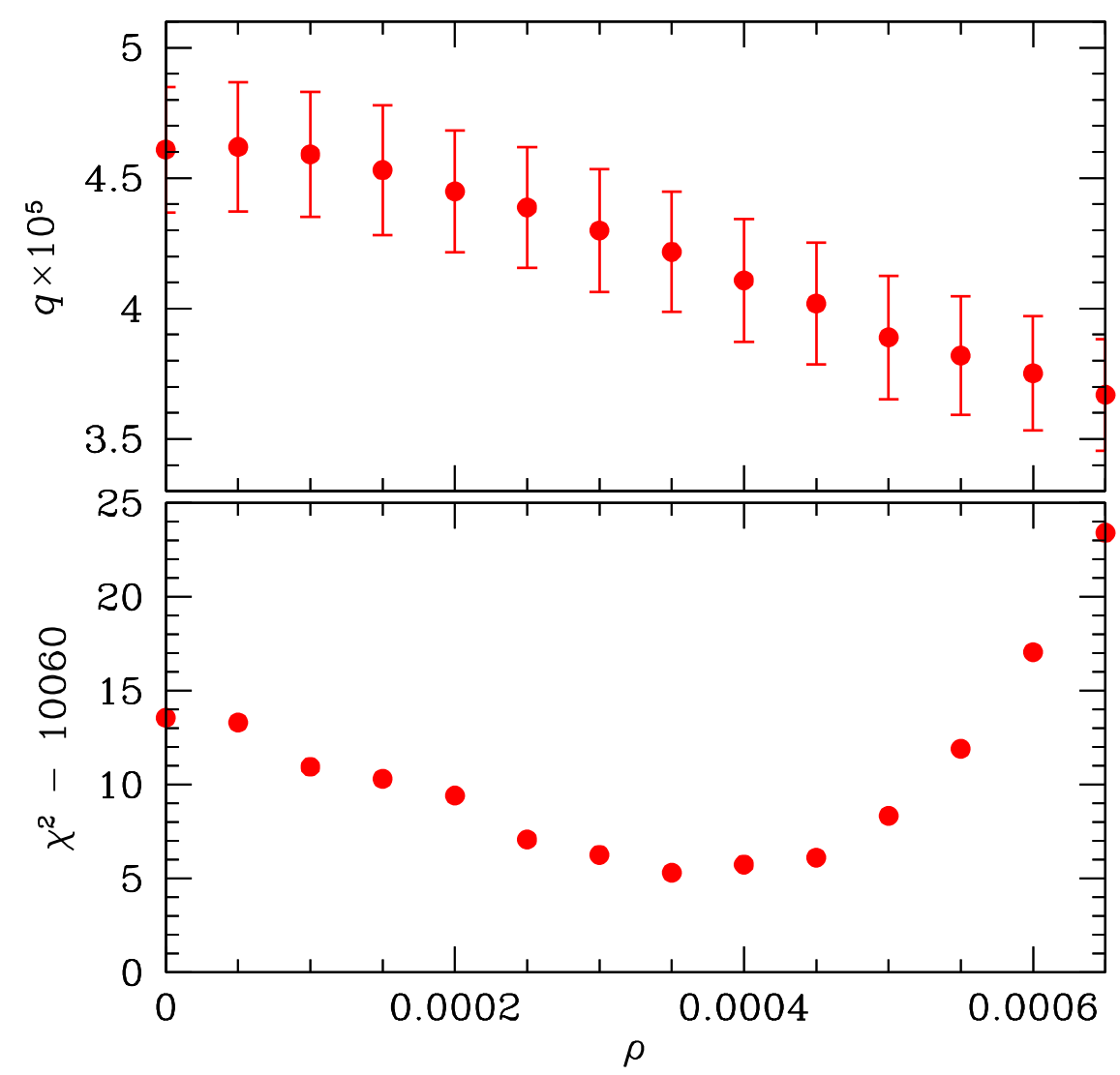

Figure 6. Lower panel: minimum values of $\chi^{2}$ for a series of MCMC runs with the normalized source size $\rho$ held fixed at the indicated values, each with 10,000 accepted elements on the chain. The minimum is overall relatively broad but high values (corresponding to small Einstein radii $\theta_{\mathrm{E}}=\theta_{*} / \rho$ ) are strongly ruled out. Upper panel: means and standard deviations of the planet-star mass ratio $q$ for each of the MCMC runs carried out to make the bottom panel. Note that $q$ is correlated with $\rho$ over the broad $\chi^{2}$ minimum of the latter. This correlation is taken into account in the Bayesian analysis (Section 5).

Table 3

Lens Properties

\begin{tabular}{lc}
\hline \hline Parameters & Values \\
\hline$q\left(10^{-5}\right)$ & $4.09_{-0.27}^{+0.26}$ \\
$\theta_{\mathrm{E}}(\mathrm{mas})$ & $1.01_{-0.15}^{+0.20}$ \\
$\mu_{\text {rel }}\left(\mathrm{mas} \mathrm{yr}^{-1}\right)$ & $8.42_{-1.24}^{+1.68}$ \\
$M_{\text {host }}\left(M_{\odot}\right)$ & $0.76_{-0.39}^{+0.42}$ \\
$M_{\text {planet }}\left(M_{\oplus}\right)$ & $10.28_{-5.28}^{+5.84}$ \\
$a_{\perp}(\mathrm{AU})$ & $3.31_{-1.47}^{+1.38}$ \\
$D_{\mathrm{L}}(\mathrm{kpc})$ & $3.32_{-1.39}^{+1.22}$ \\
\hline
\end{tabular}

mottled background results in correlated errors between the $I$ and $V$-band measurements because there can be an excess or a hole in this background of stars that is predominantly redder than the apparent blend star. Hence, an excess at the location of the event would cause the apparent blend to appear brighter and redder, while a hole in the background would make it appear fainter and bluer.

Based on the surface density of clump stars (Nataf et al. 2013; D. Nataf, 2019, private communication), we find a normalization factor of 2.41 relative to Baade's Window. We adopt $\left[E(V-I), A_{I}\right]=(1.28,1.50)$ from Nataf et al. (2013), and then evaluate the statistical errors using a 1."5 FWHM seeing disk. We find that (from this effect alone), there is a $16 \%$ probability that the blend appears at least 0.20 mag bluer than it is, as well as a $16 \%$ probability that the blend appears at least 0.28 mag redder than it is. Hence, combining the two effects (systematic and statistical errors), the color error is at least $\pm 0.28 \mathrm{mag}$.

We should also ask how well the source is astrometrically aligned with the baseline object. We measure the position of the source and baseline object relative to the KMTC42 template and find in (west, north) 0 ". 4 pixel coordinates, that the baseline object lies $(0.32,-0.12)$ pixels, or $(0$." $13,-0$ !" 05$)$, i.e., west and south of the source. Because the source position is derived from difference images (which remove both the resolved and unresolved backgrounds) and because the source is highly magnified in these images, the errors in the source position are negligible relative to the errors in the baseline object. Hence we ignore them.

The astrometric errors originate from the same three types of the photometric errors. The astrometry is done in the $I$ band, so we evaluate these errors in this band. We again only evaluate the errors of the first and third types. We estimate the fractional astrometric error (relative to the Gaussian half width $\sigma=\mathrm{FWHM} / \sqrt{\ln 256})$ as that of the fractional photometric error $(\ln 10 / 2.5) \sigma_{I}$. That is, $\sigma_{\text {ast }}=0.39 \sigma_{I}$ FWHM $\rightarrow 0$ " $6 \sigma_{I}$. We find that the photon-error contribution to $\sigma_{I}$ is 0.14 mag.

Next, we consider the error due to the mottled background. If we evaluate this without any constraint, we find $\sigma_{I}=0.6$. However, if we restrict to cases where the background produces a hole, then $\sigma_{I}=0.3$. Combined, these two sources of error imply $\sigma_{\text {ast }}=0$." 37 and $\sigma_{\text {ast }}=0$.' 20 for the two cases. This is larger than the observed offset. Hence, the measured astrometric offset is not inconsistent with the hypothesis that 

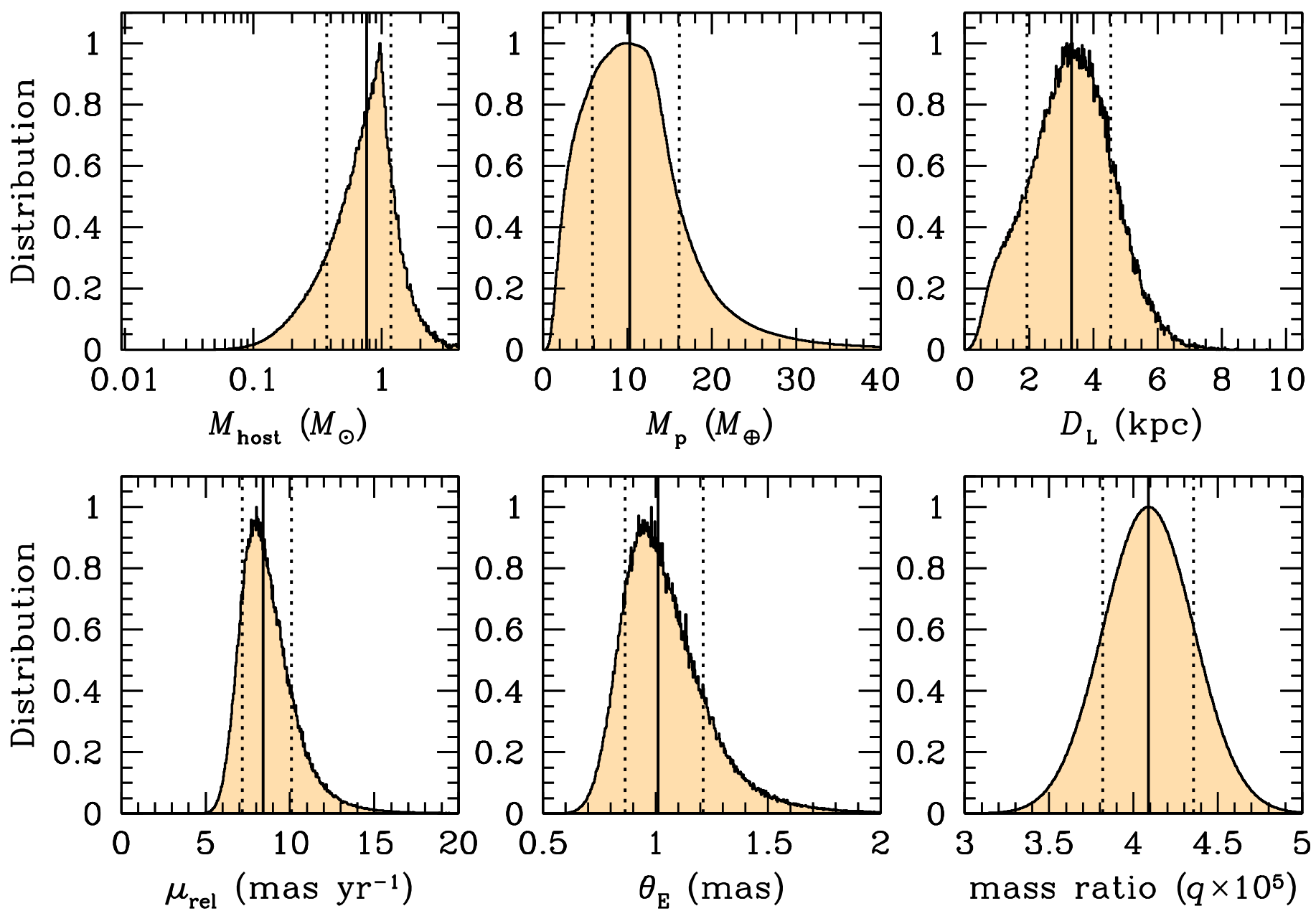

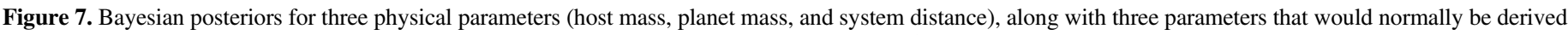

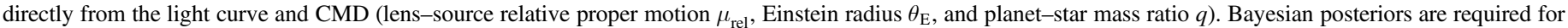
these three because $\rho$ is relatively poorly determined and with significantly asymmetric errors. In particular, $q$ is correlated with $\rho$ (see Figure 6).

the blend is the lens. However, we note that this hypothesis can be established by future high-resolution observations.

\section{Discussion}

\subsection{Very Low Mass-ratio Planets}

It is found that KMT-2019-BLG-0842Lb is a cold planet located beyond the snow line of its host, and the planet/host mass ratio is $q=(4.09 \pm 0.27) \times 10^{-5}$, which is similar to the ratio of Uranus/Sun in the solar system. The discovery of the planetary system, together with similar systems previously discovered, provides evidence that such planets are not rare. Nevertheless, further discoveries will be necessary to estimate the frequency and characterize the distribution of such planets.

As noted by Ryu et al. (2020), the detection pace of lowmass-ratio microlensing planets with $q \leqslant 1 \times 10^{-4}$ has been accelerating since 2015, when KMTNet commenced. The 11 such planets (including KMT-2018-BLG-0029Lb and KMT2019-BLG-0842Lb) occurred in 2005, 2005, 2007, 2009, 2013, 2015, 2016, 2017, 2018, 2018, and 2019. That is, five during the 10 seasons before 2015 and six during the five seasons after. Moreover, the data from the 2019 season have not yet been fully analyzed. If indeed sub-Uranus/Sun planets are common, we can expect more detections based on the trend of increasing rate.
In this context, it is worthwhile to ask what features of low mass-ratio planetary events allow them to be detected. A related issue is how the planet detection strategy should be adjusted to maximize the detection rate.

A notable feature of KMT-2019-BLG-0842Lb is that the planetary signal appeared in a high-magnification event $\left(u_{0}=\right.$ $\left.0.0066 ; A_{\max }=150\right)$, and at very low angle $\alpha=0.146\left(8^{\circ} .5\right)$ between the source trajectory and the binary axis. That is, the magnification of the underlying $1 \mathrm{~L} 1 \mathrm{~S}$ event at the time of the planetary anomaly was modest: $A_{\text {anom }} \simeq \sin (\alpha) / u_{0}=22$. This means that the source would have passed over the same part of the caustic structure if the event had had $A_{\max }=$ $22\left(u_{0}=0.045\right)$ and $\alpha=90^{\circ}$. This is similar to the situations for OGLE-2016-BLG-1195, which had $\left|u_{0}\right|=0.053,|\alpha|=$ $55^{\circ}$, and a slightly higher mass ratio, and for KMT-2018-BLG0029 , which had $\left|u_{0}\right|=0.027,|\alpha|=88^{\circ}$, and a substantially lower mass ratio. Thus, at first sight, it seems that such moderate-magnification events provide as fertile ground to hunt for low-mass planets as high-magnification events (as advocated by Abe et al. 2013). In fact, however, KMT-2019-BLG0842 Lb would have been discovered for values of $\alpha$ covering most of the unit circle at its actual $u_{0}$, whereas at $u_{0}=0.045$ the sensitivity would have been restricted to a relatively narrow range of angles. 
However, the main feature to be noted is that the low sourcetrajectory angle caused the anomaly to be longer by a factor of $\cot \alpha \simeq 7$ relative to an orthogonal transit of the caustic structure. That is, in an orthogonal crossing, the full duration would have been about two hours rather than 15 hours. This is just twice the source-diameter self-crossing time, $2 t_{*} \equiv 2 \rho t_{\mathrm{E}}=0.9 \mathrm{hr}$. Such a short anomaly would have been detected in the actual observations (provided that it did not fall in a gap) because the event was in a high-cadence KMT field, with $\Gamma=4-6 \mathrm{hr}^{-1}$. But if it had been in a field with a cadence of $\Gamma=0.75-1 \mathrm{hr}^{-1}$, then a twohour anomaly would have been missed, while a 15-hour anomaly (as in this case) can be readily detected.

These points are in some sense moot because the survey strategy is basically set. However, these anomalies are not necessarily noticed in the mode by which events are currently vetted, i.e., either manual or machine review of pipeline data, with optimized photometry only for those events that look interesting. The case of KMT-2019-BLG-0842 demonstrates that very low- $q$ planets can give rise to long-lived ( $~(10 \mathrm{hr}$ ) low-amplitude $(\lesssim 0.1 \mathrm{mag})$ bumps several magnitudes below the peak of high-magnification events. Such planetary signals can be missed by the present search process. Hence, it would seem to be prudent to make optimized photometry of all highmagnitude events.

Moreover, there is a separate question as to how to marshal follow-up observations in order to probe to the lowest massratio planets possible, i.e., planets of substantially smaller $q$ than those that have been detected to date. At present, the rule of thumb is to intensively monitor high-magnification events within the FWHM around $t_{0}$ because this region contains most of the sensitivity to planets. Specifically, "most" translates to a fraction of $\sim\left(\sec ^{-1} 2\right) /(\pi / 2)=2 / 3$. However, planets like KMT-2019-BLG-0842Lb lie in the other (remaining) one-third of the circle. Although a minority, these planets can give rise to long-lived perturbations even when the planet has a low mass ratio $q$, which can be of exceptional interest. Thus, particularly in very high-magnification events, for which $t_{\mathrm{eff}}$ is short, follow-up observations during $t_{\text {eff }}$ are less expensive to carry out. Therefore, follow-up observations should be more aggressively pursued for very high-magnification events.

\subsection{Future High-resolution Imaging and Spectroscopy}

As discussed in Section 5.1, both the photometry and astrometry indicates the possibility that most of the blended light is generated by the lens. This possibility can be tested with high-resolution imaging, either with Hubble Space Telescope (HST) or ground-based adaptive optics (AO) mounted on very large ground-based telescopes. Based on the Bayesian estimates together with the star catalog of Pecaut \& Mamajek (2013), we estimate the dereddened lens magnitude in the $V, I$, and $H$ band as $(V, I, H)_{\mathrm{L}, 0}=\left(18.78_{-1.90}^{+4.95}, 17.86_{-1.62}^{+3.45}, 16.75_{-1.19}^{+2.63}\right)$. For the source, we estimate the dereddened magnitude as $(V, I, H)_{\mathrm{S}, 0}=$ $(21.14 \pm 0.07,20.13 \pm 0.04,18.85 \pm 0.07)$ based on our CMD analysis. These imply that the blended light is $\sim 2$ mag brighter than the source in the $I$ band. Therefore, if HST I-band images show that this blended light is closely aligned with the source position, then this will provide strong evidence that the blended light is due to the lens. In principle, such aligned light may originate from a stellar companion to either the source or the lens. However, if the alignment is very close (10-20 mas), then this will rule out the possibility of the lens companion because such a companion would have generated significant deviations over the well-covered peak of the event. The possibility of the source companion can ultimately be ruled out by re-imaging the field when the source and blend are separated far enough to measure their relative proper motion. Because the source and blend have substantially different colors, such a measurement does not require the two stars to be separately resolved, but can be carried out via a measurement of the astrometric offset between their combined light in different bands (Bennett et al. 2006).

Ground-based AO astrometry will also be useful to check whether the blended light is aligned with the lens. The excess flux from the blend will be somewhat more difficult to measure in this case because such measurements will be conducted in near-infrared bands (e.g., the $H$ band), for which there is no direct measurement of the source flux. Nevertheless, based on VIH color-color relations of nearby field stars, derived from the KMTC42 CMD (Figure 5) and the future AO measurements aligned to standard catalogs, it should be possible to predict the $H$-band source flux with reasonably good precision. Even though the blended light is much bluer than the source, it should still be substantially brighter in the $H$ band than the source (because it is $2 \mathrm{mag}$ brighter in the $I$ band). Thus, ground-based AO measurements should be feasible.

The Bayesian posterior estimate of the lens-source relative

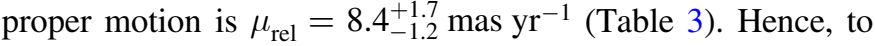
obtain the first measurement when the lens and source are still closely aligned, the observations should be made in 2020 , if possible.

If the blended light proves to be aligned with the source, then it should be possible to spectroscopically classify the blend (Han et al. 2019). Such an observation may also plausibly measure the radial velocity (RV) offset between the source and the blend. If so, this will provide a second (and earlier) method to rule out the blend as being a companion to the source. Note that, to avoid generating a noticeable bump on the light curve, the blend (as companion) must have a projected separation $\gtrsim 8 \mathrm{au}$, and so an $\mathrm{RV}$ offset of $\lesssim 15 \mathrm{~km} \mathrm{~s}^{-1}$ relative to the source.

If the blend proves not to be the lens (or, more precisely, not to be dominated by the lens light), then it should still be possible to characterize the lens with high-resolution follow-up. Depending on the flux and/or color differences between the source and the lens, this may be achieved even before the images are fully separated, e.g., by measuring the astrometric offset between the combined images in different bands (Bennett et al. 2006) or by measuring the elongation of the combined image (Bhattacharya et al. 2018). Even if the flux ratio is extreme, causing these combined-image techniques to fail, the lens can still be separately imaged when it has moved $\sim 60$ mas (Batista et al. 2015) from the source, i.e., roughly by 2027. From Figure 7, the lens mass is almost certainly above the hydrogen-burning limit, so this method is almost guaranteed to work if all others fail.

W.Z. acknowledges support by the National Science Foundation of China (grant No. 11821303 and 11761131004) Work by A.G. was supported by AST-1516842 from the US NSF and by JPL grant 1500811. A.G. received support from the European Research Council under the European Union's Seventh Framework Programme (FP 7) ERC grant Agreement No. [321035] This research has made use of the KMTNet system operated by the Korea Astronomy and Space Science Institute (KASI) and the 
data were obtained at three host sites of CTIO in Chile, SAAO in South Africa, and SSO in Australia. Work by C.H. was supported by the grants of National Research Foundation of Korea (2017R1A4A1015178 and 2020R1A4A2002885). The OGLE project has received funding from the National Science Centre, Poland, grant MAESTRO 2014/14/A/ST9/00121 to A.U. The MOA project is supported by JSPS KAKENHI grant No. JSPS24253004, JSPS26247023, JSPS23340064, JSPS15H00781, and JP16H06287. Work by C.R. was supported by an appointment to the NASA Postdoctoral Program at the Goddard Space Flight Center, administered by USRA through a contract with NASA.

\section{ORCID iDs}

Youn Kil Jung (iD https://orcid.org/0000-0002-0314-6000 Andrzej Udalski (iD https://orcid.org/0000-0001-5207-5619 Jennifer C. Yee (iD https://orcid.org/0000-0001-9481-7123 Cheongho Han (D) https://orcid.org/0000-0002-2641-9964 Michael D. Albrow (D) https://orcid.org/0000-0003-3316-4012 Sun-Ju Chung (i) https://orcid.org/0000-0001-6285-4528 Kyu-Ha Hwang (ib https://orcid.org/0000-0002-9241-4117 Yoon-Hyun Ryu (iD https://orcid.org/0000-0001-9823-2907 In-Gu Shin (ib https://orcid.org/0000-0002-4355-9838 Yossi Shvartzvald (ib https://orcid.org/0000-0003-1525-5041 Hyoun-Woo Kim (iD https://orcid.org/0000-0001-8263-1006 Seung-Lee Kim (iD https://orcid.org/0000-0003-0562-5643 Chung-Uk Lee (1) https://orcid.org/0000-0003-0043-3925

Richard W. Pogge (ib https://orcid.org/0000-0003-1435-3053

Przemek Mróz (10) https://orcid.org/0000-0001-7016-1692 Michał K. Szymański (i) https://orcid.org/0000-00020548-8995

Jan Skowron (iD https://orcid.org/0000-0002-2335-1730 Igor Soszyński (i) https://orcid.org/0000-0002-7777-0842 Paweł Pietrukowicz (i) https://orcid.org/0000-0002-2339-5899 Krzysztof Ulaczyk (i) https://orcid.org/0000-0001-6364-408X Patryk Iwanek (i) https://orcid.org/0000-0002-6212-7221 Richard Barry (iD https://orcid.org/0000-0003-4916-0892 David P. Bennett (1) https://orcid.org/0000-0001-8043-8413 Akihiko Fukui (i) https://orcid.org/0000-0002-4909-5763 Yoshitaka Itow (iD https://orcid.org/0000-0002-8198-1968 Iona Kondo (ib https://orcid.org/0000-0002-3401-1029 Naoki Koshimoto (iD https://orcid.org/0000-0003-2302-9562 Shota Miyazaki (iD https://orcid.org/0000-0001-9818-1513 Clément Ranc (iD https://orcid.org/0000-0003-2388-4534
Nicholas J. Rattenbury (iD https://orcid.org/0000-00015069-319X

Daisuke Suzuki (iD https://orcid.org/0000-0002-5843-9433

\section{References}

Abe, F., Airey, C., Barnard, E., et al. 2013, MNRAS, 431, 2975

Alard, C. 2000, A\&AS, 144, 363

Alard, C., \& Lupton, R. H. 1998, ApJ, 503, 325

Albrow, M. D., Horne, K., Bramich, D. M., et al. 2009, MNRAS, 397, 2099

Batista, V., Beaulieu, J.-P., Bennett, D. P., et al. 2015, ApJ, 808, 170 Beaulieu, J.-P., Bennett, D. P., Fouqué, P., et al. 2006, Natur, 439, 437

Bennett, D. P., Anderson, J., Bond, I. A., Udalski, A., \& Gould, A. 2006, ApJL, 647, L171

Bennett, D. P., \& Rhie, S.-H. 1996, ApJ, 472, 660

Bensby, T., Yee, J. C., Feltzing, S., et al. 2013, A\&A, 549A, 147

Bessell, M. S., \& Brett, J. M. 1988, PASP, 100, 1134

Bhattacharya, A., Beaulieu, J.-P., Bennett, D. P., et al. 2018, AJ, 156, 289

Bond, I. A., Bennett, D. P., Sumi, T., et al. 2017, MNRAS, 469, 2434

Bond, I. A., Udalski, A., Jaroszyński, M., et al. 2004, ApJL, 606, L155

Gaudi, B. S. 1998, ApJ, 506, 533

Gould, A. 1992, ApJ, 392, 442

Gould, A. 2000, ApJ, 542, 785

Gould, A. 2004, ApJL, 606, 319

Gould, A., \& Loeb, A. 1992, ApJ, 396, 104

Gould, A., Ryu, Y.-H., Calchi Novati, S., et al. 2020, JKAS, 53, 9

Gould, A., Udalski, A., An, D., et al. 2006, ApJL, 644, L37

Han, C., Yee, J. C., Udalski, A., et al. 2019, AJ, 158, 102

Holtzman, J. A., Watson, A. M., Baum, W. A., et al. 1998, AJ, 115, 1946

Hwang, K.-H., Udalski, A., Shvartzvald, Y., et al. 2018, AJ, 155, 20

Jung, Y. K., Gould, A., Zang, W., et al. 2019, AJ, 157, 72

Jung, Y. K., Udalski, A., Gould, A., et al. 2018, AJ, 155, 219

Kervella, P., Thévenin, F., Di Folco, E., \& Ségransan, D. 2004, A\&A, 426, 297

Kim, D.-J., Kim, H.-W., Hwang, K.-H., et al. 2018b, AJ, 155, 76

Kim, H.-W., Hwang, K.-H., Kim, D.-J., et al. 2018c, AJ, 155, 186

Kim, H.-W., Hwang, K.-H., Kim, D.-J., et al. 2018d, arXiv:1804.03352

Kim, H.-W., Hwang, K.-H., Shvartzvald, Y., et al. 2018a, arXiv:1806.07545

Kim, S.-L., Lee, C.-U., Park, B.-G., et al. 2016, JKAS, 49, 37

Nataf, D. M., Gould, A., Fouqué, P., et al. 2013, ApJ, 769, 88

Paczyński, B. 1986, ApJ, 304, 1

Pecaut, M. J., \& Mamajek, E. E. 2013, ApJS, 208, 9

Ryu, Y.-H., Udalski, A., Yee, J. C., et al. 2020, AJ, 160, 183

Shvartzvald, Y., Yee, J. C., Calchi Novati, S., et al. 2017, ApJL, 840, L3

Suzuki, D., Bennett, D. P., Sumi, T., et al. 2016, ApJ, 833, 145

Szymanski, M., Udalski, A., Soszyński, I., et al. 2011, AcA, 61, 83

Tomaney, A. B., \& Crotts, A. P. S. 1996, AJ, 112, 2872

Udalski, A., Ryu, Y.-H., Sajadian, S., et al. 2018, AcA, 68, 1

Udalski, A., Szymanski, M. K., Soszynski, I., \& Poleski, R. 2008, AcA, 58, 69

Udalski, A., Szymanski, M. K., Szymanski, G., et al. 2015, AcA, 65, 1

Woźniak, P. R. 2000, AcA, 50, 421

Yoo, J., DePoy, D. L., Gal-Yam, A., et al. 2004, ApJ, 603, 139 